\title{
Immunotherapy in Gastrointestinal Carcinoma - How to Separate Hope from Hype
}

\author{
Markus Moehler \\ University Medical Center of the Johannes Gutenberg University Mainz, Mainz, Germany
}

\section{Dear Colleagues,}

On behalf of all authors, it is my great pleasure to present you with this mini-review series on the most current achievements and challenges in immunotherapy of various gastrointestinal (GI) cancers.

Recently, some checkpoint inhibitors have been approved for cancer treatment, which opens new treatment options and provides excellent perspectives for highly immunogenic tumors. Blocking of the B7 immunoglobulin superfamily molecules, such as PD-1 (nivolumab, pembrolizumab), CTLA-4 (tremelimumab, ipilimumab) or PD-L1 (durvalumab, atezolizumab, avelumab), has already shown promising results in many trials in patients with advanced or metastatic GI malignancies. These trials and further upcoming studies are described in more detail in the articles of this review series [1-8].

As GI tumors are very heterogeneous, there is a strong need to define efficacy in the different consensus molecular subtypes (CMS) $[1,2]$. Additionally, it is of scientific significance to increase our understanding of the interactions between tumor microenvironment and the immune system to generate more effective therapies, particularly in non-responsive tumors [3].

The challenge for the future will be to identify those patients that are most likely to be responsive to immunotherapy. Cancers characterized by microsatellite instability (MSI) or DNA damage repair deficiency are the best examples for immune-responsive tumors. Inflammation-induced tumors, such as Epstein-Barr virus (EBV)-positive tumors may also be good models for immune-responsive cancers. Despite the presented accomplishments [1-8], we still lack well-defined selection biomarkers for immunotherapy of the large patient groups with chromosomally instable and stable cancers. The search to switch these mostly non-immunogenic, socalled cold, tumors into hot immune-responsive tumors has become an exciting clinical and translational research field.

Overall, 98\% of adults worldwide are infected with EBV. The virus remains in the body of the host for a lifetime. Most of the time, the infection is asymptomatic. In intermittent cases, the emergence of lymphoma, gastric, or head and neck carcinoma is evoked. Typically, the EBV lytic replication (the process that generates new virus paricles) is a strong risk factor for these cancer types. Most recently, a new mechanism was identified by which EBV particles can induce chromosomal instability without establishing a chronic infection, thereby conferring a risk for development of tumors that do not necessarily carry the viral genome. In detail, the EBV viral protein BNRF1 induced centrosome amplification, but BNRF1-deficient viruses largely lost this property [9]. - Do we have here a new marker for chromosomal instability and potential immunogenic tumors that is not yet known?

All in all, there are exciting times ahead for us as clinical scientists and as practitioners. As we were able to show in our publications, the immune system is a rather gifted therapeutic partner in combination with chemotherapy, irradiation, or other innovative strategies. We have great hopes for new immunotherapy combinations with classical treatment strategies - even if we might not yet fulfil every patient's expectations. In the last article of this series [8], we discuss tumor associated macrophages (TAM); myeloid derived suppressor cells (MDSC) as well as regulatory T cells (Tregs) as new immunotherapeutic targets. In addition, we describe how blockade of the GITR, IDO, Csf1R, or WNT signaling pathways offers capable approaches for overcoming the immune silencing within the non-responsive or resistant tumor microenvironment.

The meticulous assessment of the genetic background and our biomarker panels will help us to separate real hope from hype. These new studies give us a common challenge and new perspectives for our patients.

\section{Disclosure Statement}

Markus Möhler acknowledges receiving the following: grants/research support: AIO, Amgen, BMS, EORTC, Merck, MSD, Taiho, Roche; honoraria or consultation fees: Amgen, AstraZeneca, BMS, Falk, Lilly, MCI, Merck, MSD, Nordic, Pfizer, Roche, Servier.

\section{KARGER}

(๑) 2018 S. Karger GmbH, Freiburg
Prof. Dr. med. Markus Moehler 


\section{References}

1 Stein A, Folprecht G: Immunotherapy of colon cancer. Oncol Res Treat 2018;41 DOI: 10.1159/000488918.

2 Smyth E, Thuss-Patience P: Immunotherapy of gastric cancer. Oncol Res Treat 2018;41 DOI: 10.1159/ 000489099.

3 Cheung PF, Lutz M, Siveke JT: Immunotherapy and combination strategies in pancreatic cancer: curren status and emerging trends. Oncol Res Treat 2018;41 DOI: $10.1159 / 000488917$.
4 Alsina M, Moehler M, Lorenzen S: Immunotherapy of esophageal cancer: current status, many trials and innovative strategies. Oncol Res Treat 2018;41 DOI: 10.1159/000488120.

5 Heinrich B, Czauderna C, Marquardt JU: Immunotherapy of hepatocellular carcinoma. Oncol Res Treat 2018;41 DOI: 10.1159/000488916.

6 Tariq N, Vogel A, McNamara, Valle JW: Biliary tract cancer: implicated immune-mediated pathways and their associated potential targets. Oncol Res Treat 2018;41 DOI: 10.1159/000488997.
7 Weber MM, Fottner C: Immune checkpoint inhibitors in the treatment of patients with neuroendocrine neoplasia. Oncol Res Treat 2018;41 DOI: 10.1159/ 000488996.

8 Moehler M, Göpfert K, Lenz HJ: Outlook: immunotherapy in gastrointestinal carcinoma innovative strategies. Oncol Res Treat 2018;41 DOI: 10.1159/000489047.

9 Shumilov A, Tsai MH, Schlosser YT, et al.: EpsteinBarr virus particles induce centrosome amplification and chromosomal instability. Nat Commun 2017;8: 14257. 